\title{
A DIMENSÃO SOCIAL DA RESPONSABILIDADE PESSOAL
}

\author{
Valdiney Veloso Gouveia* \\ Tatiana Cristina Vasconcelos \\ Fabiana Queiroga" \\ Maria Luiza Pontes de França ${ }^{\text {E }}$ \\ Suenny Fonseca de Oliveira ${ }^{\infty}$
}

\begin{abstract}
RESUMO. A responsabilidade pessoal é um construto importante para compreender o comportamento das pessoas no cotidiano; portanto, mereceria atenção dos pesquisadores. A escassez de informação a respeito motivou a presente pesquisa, cujo objetivo foi conhecer em que medida a responsabilidade pessoal se correlaciona com os valores e o individualismocoletivismo apresentados pelas pessoas. Participaram 250 estudantes universitários de diferentes áreas do conhecimento, sendo a maioria do sexo feminino $(82 \%)$, com idade média de 20 anos $(\mathrm{DP}=2,94)$. Estes responderam a três instrumentos: Escala de Responsabilidade Pessoal, Questionário dos Valores Básicos e Questionário de Individualismo-Coletivismo, além de perguntas demográficas. Como esperado, a responsabilidade pessoal se correlacionou diretamente com os valores sociais, tendo feito inversamente com os pessoais e o índice de individualismo. Concluiu-se que a dimensão da responsabilidade pessoal tem uma natureza eminentemente social, principalmente em culturas como a espanhola, que dá ênfase às relações interpessoais. Sugere-se a replicação deste estudo no Brasil.
\end{abstract}

Palavras-chave: responsabilidade pessoal, individualismo, valores sociais.

\section{THE SOCIAL DIMENSION OF THE PERSONAL RESPONSIBILITY}

\begin{abstract}
The social responsibility is an important construct to understand people's daily behavior; therefore it should attract the researchers' attention. A lack of information about the topic motivated the present research, which aimed at knowing at what level the personal responsibility correlates with values and individualism-collectivism expressed by people. Took part in this research, a sample of 250 university students from different areas of knowledge, mainly females (82\%), average of 20 years of age $(\mathrm{SD}=2.94)$. These students answered three instruments: Personal Responsibility Scale, Basic Values Questionnaire and Individualism-Collectivism Questionnaire, with additional demographic questions. As expected, the personal responsibility is directly correlated with the social values and negatively with personal values and individualism index. The research concluded that personal responsibility dimension has a social nature, especially in cultures that emphasize the interpersonal relations, as the Spanish culture. It is suggested the replication of this study in Brazil.
\end{abstract}

Key words: personal responsibility, individualism, social values.

\section{INTRODUÇÃO}

O homem é definido como um ser social, que atua segundo normas, interage e compartilha direitos e deveres na sociedade. Neste sentido, ele está sendo constantemente questionado sobre sua conduta em relação aos demais e cobrado a apresentar responsabilidade perante suas ações (Gouveia,
Clemente, Vidal \& Martínez, 2000). Parece evidente a importância de estudar a responsabilidade; isso permitiria definir estratégias que objetivassem sua promoção, evitando condutas socialmente indesejáveis, como a corrupção, por exemplo. Não obstante, este tem sido um tema que carece de estudos empíricos dentro da Psicologia. Tal aspecto motivou a presente pesquisa, que visa conhecer os antecedentes

\footnotetext{
* Doutor em Psicologia Social pela Universidade Complutense de Madri, Professor da Universidade Federal da Paraíba.

\# Mestranda da Universidade Federal da Paraíba.

II Psicóloga Universidade Federal da Paraíba.

Æ Bolsista de Iniciação Científica, Acadêmica do curso de Psicologia da Universidade Federal da Paraíba.

x Acadêmica do curso de Psicologia da Universidade Federal da Paraíba.
} 
sociais da responsabilidade pessoal. Especificamente, dar-se-á ênfase a dois construtos que potencialmente podem ajudar a explicá-la, como são os valores humanos (Rokeach, 1973) e o individualismocoletivismo (Waterman, 1981). Portanto, faz-se necessário considerar mais detidamente tais construtos, o que se fará no decorrer deste texto; antes, porém, apresenta-se a seguir a conceituação de responsabilidade.

\section{O CONCEITO DE RESPONSABILIDADE}

Ao se considerar o termo responsabilidade, especificamente na Psicologia Social, observa-se que os estudos a respeito centram-se em dois enfoques diferenciados, a saber: responsabilidade social e responsabilidade pessoal. A primeira significa "atuar sem buscar benefícios para si ou para as pessoas do seu grupo de pertença, independente das conseqüências que isso possa ter" (Gouveia \& Alves, 1993, p. 50). Este tipo de responsabilidade implica necessariamente uma interação com outros, seja esta real ou imaginária. Neste contexto, como têm revelado alguns estudos (O’Neill \& Eisner, 1981; Sigelmann \& Fernandes, 1984), as pessoas buscam se ajustar às normas estabelecidas por seus grupos de pertença, procurando a aprovação dos membros destes, ao mesmo tempo em que buscam manter uma imagem positiva diante de si e de outras pessoas, as quais poderiam ser definidas como avaliadoras potenciais de seu comportamento.

A responsabilidade pessoal, foco principal deste estudo, compreende uma dimensão da responsabilidade social e está subjacente em teorias clássicas da Psicologia Social, como a da atribuição de causalidade (Gouveia e cols., 2000). Contempla a noção de um indivíduo cumpridor daquilo que se propõe. Segundo Waterman (1981), é um compromisso primeiro consigo, com independência das normas sociais. De acordo com esta perspectiva, que reconhecidamente se pauta em uma visão eminentemente individualista, na responsabilidade pessoal, ainda que exista influência daquilo que é considerado socialmente aceito, o ator age segundo o que ele determina como meta ou se compromete a fazer. Portanto, esperar-se-ia que as pessoas que apresentassem responsabilidade pessoal se orientassem por valores que pusessem ênfase na liberdade do indivíduo.

A responsabilidade pessoal pode ser também resultado do contexto sociocultural em que estão inseridas as pessoas. Embora possa ser mais preponderante $\mathrm{o}$ sentido de independência e liberdade na determinação da responsabilidade pessoal em culturas individualistas, talvez isso não ocorra naquelas culturas mais tendentes ao coletivismo, como é o caso do Brasil (Gouveia, Albuquerque, Clemente \& Espinosa, 2003), ou naquelas que dão máxima importância às relações interpessoais, como ocorre com a Espanha (Smith, Dugan \& Trompenaars, 1996). Nestas culturas, em função da dependência existente entre membros dos grupos, o sentido de obrigação e apego emocional com os demais pode configurar uma maior responsabilidade pessoal daqueles que agem de acordo com estes indicadores, refletidos nos valores que guiam suas vidas. Não obstante, esta é uma hipótese que precisará ser comprovada. Cabe neste instante conhecer mais acerca das orientações individualista e coletivista, como abaixo tratadas.

\section{A DIMENSÃO INDIVIDUALISMO- COLETIVISMO}

O individualismo-coletivismo surgiu inicialmente como uma dimensão cultural. Compreendia então uma orientação valorativa, que permitia comparar os países, os quais eram classificados como individualistas -por exemplo, os Estados Unidos, a Austrália) ou coletivistas por exemplo, o Paquistão, a Venezuela) (ver Hofstede, 1984). Porém, prontamente passou a ser considerado como um construto psicológico, dando origem a um programa de pesquisa transcultural na Psicologia. Apesar da sua origem antropológica ou sociológica, foi com os psicólogos que esta dimensão se fez mais popular. Especificamente, $\mathrm{H}$. C. Triandis constitui um nome de referência sobre este tema, tendo apresentado ao menos 66 hipóteses acerca dos seus antecedentes e conseqüentes (Triandis, 1994, 1995). A título de organização do texto, é possível definir sumariamente estes construtos, como segue (Gouveia e cols., 2003):

- Individualismo: enfatiza a individualidade, tratada em termos de independência das demais pessoas e dos grupos. Dá ênfase principalmente ao êxito; o indivíduo é considerado como a unidade principal de sobrevivência, sobrepondo-se em geral aos grupos. Neste tipo de orientação, as relações pessoais podem ser mais freqüientes, porém apresentam uma natureza contratual. Claramente se evidencia uma separação do indivíduo em relação aos seus familiares e uma ruptura com os ancestrais (Gouveia, Clemente \& Vidal, 1998). Apesar de este poder se definir como membro de muitos grupos, na 
realidade tais grupos não são aqueles de pertença; este tipo de grupo está restringido a sua família nuclear. A pessoa individualista age segundo seus próprios interesses, dando pouca importância ao contexto social em que se encontra.

- Coletivismo: este tipo de orientação ressalta principalmente o cumprimento das obrigações com os demais, no sentido de seguir a orientação do grupo de pertença. Este é considerado mais importante do que a própria pessoa em si. Apesar de poderem ser mais reduzidos, os grupos de pertença são geralmente grandes em extensão (por exemplo, a família com todos os seus membros). Dentro dos grupos os indivíduos mantêm fortes relações entre si, compartilhando geralmente os mesmos interesses (Gouveia, Clemente \& Vidal, 1998). Cada pessoa dentro do grupo é valorizada como um fim em si; o indivíduo atua levando em consideração o contexto e as demais pessoas com as quais compartilha o sentido de pertença grupal.

Ainda que possam ser definidos separadamente, ao menos na presente pesquisa estes construtos são considerados como componentes de uma dimensão bipolar de orientação social, descrevendo o apego (coletivismo) vs a independência (individualismo) dos grupos. Neste sentido, esperar-se-ia que as pessoas individualistas se caracterizassem por valorizar a independência emocional do indivíduo em relação aos grupos sociais; já as coletivistas valorizariam a tradição herdada e a dependência emocional dos grupos, pois o indivíduo é definido como um ser que adquire sentido como parte de uma coletividade. Esta concepção, apesar de ser algo simplista, reflete duas tendências básicas, apresentadas em diferentes modelos e tipologias de orientação social (Gouveia, 1998; Triandis, 1995).

Em resumo, considerando-se sua condição de orientar a conduta social (Triandis, 1994), o individualismo-coletivismo apresentado pelas pessoas pode ter relação com a responsabilidade pessoal que estas manifestam. Esta dimensão também se relaciona com os valores humanos apresentados pelas pessoas, embora não com todos. Por exemplo, Gouveia, Andrade, Milfont, Queiroga e Santos (2003), em uma amostra de 304 participantes, entre membros da população geral e estudantes universitários e do ensino médio, observaram que os valores autodireção, beleza, justiça social e ordem social não se correlacionaram com o individualismo e o coletivismo. Estes aspectos permitem pensar que tais construtos não são isomorfos, sugerindo que sejam considerados separadamente. Considerar-se-á a seguir o modelo dos valores humanos que será tomado como referência na presente pesquisa.

\section{A TEORIA DOS VALORES HUMANOS BÁSICOS}

Existem diferentes modelos teóricos sobre os valores humanos (Braithwaite \& Scott, 1991). Não obstante, parece se configurar como uma abordagem bastante consolidada a que assume os valores como representação das necessidades humanas (Rokeach, 1973; Schwartz, 1994). Dentro desta perspectiva, teve origem a teoria dos valores básicos (Gouveia, 1998), que configura o marco de referência do presente estudo. Este modelo define os valores como representação das necessidades e das pré-condições para satisfazê-las, segunda a concepção de Maslow (1954). Em resumo, a tipologia em pauta estima a existência de três critérios de orientação valorativa, os quais dão origem a seis funções psicossociais, como segue: pessoal (experimentação e realização), central (existência e suprapessoal) e social (interacional e normativa). Uma definição sumária destes é apresentada a seguir:

- Valores pessoais: quem os adota dá prioridade aos próprios benefícios. Estes são valores de foco intrapessoal, centrados na própria pessoa. Dividemse em duas funções psicossociais: (a) experimentação: ressalta descobrir e apreciar novos estímulos, enfrentar situações-limite, assim como buscar satisfação sexual (emoção, estimulação sexual); e (b) realização: acentua a realização, o sentimento de ser importante e poderoso, ser uma pessoa com identidade e espaço próprio. Quem assume este padrão de valores costuma manter relações pessoais contratuais, com o fim de obter benefícios (autodireção, êxito, poder, prestígio e privacidade).

- Valores centrais: tais valores são compatíveis com os valores pessoais e sociais. Suas funções psicossociais são: (a) existência: expressa uma preocupação por garantir a própria existência orgânica (estabilidade pessoal, saúde e sobrevivência); a ênfase não é centrada sobre a individualidade pessoal, mas sobre a existência individual; e (b) suprapessoal: define a situação social por excelência em que a pessoa pode obter seus objetivos independentemente de sua condição social ou grupo afiliativo; descreve alguém maduro, com preocupações menos materiais (beleza, conhecimento, justiça social e maturidade). Os valores desta função enfatizam a importância de todas as pessoas, não exclusivamente dos indivíduos que compõem o grupo de pertença. 
- Valores sociais: quem assume estes valores se orienta em direção aos demais, manifestando o desejo de se sentir considerado e buscando assegurar sua aceitação e integração no seu grupo. Esta orientação de valores se divide nas seguintes funções psicossociais: (a) normativa: ênfase na vida social, na busca de estabilidade do grupo, nos comportamentos socialmente corretos e no respeito pelos símbolos e padrões culturais que prevaleceram durante anos; estimase a ordem acima de qualquer outra coisa (ordem social e religiosidade e tradição); e (b) interacional: focaliza-se a atenção nas demais pessoas. Sua especificidade enquanto conjunto fundamenta-se no interesse por se sentir querido, ter amizades verdadeiras e uma vida social (afetividade, apoio social, convivência e honestidade).

Em resumo, este modelo dos valores humanos tem sido comprovado em outros estudos;. Gouveia (1998) o testou em três amostras brasileiras (Distrito Federal, Paraíba e São Paulo) e três espanholas (Barcelona, Madri e Pontevedra). Recentemente, Gouveia e cols. (2002) comprovaram sua utilidade para explicar a identificação com grupos sociais e unidades geoespaciais (por exemplo, cidade, estado etc.).

Tendo apresentado o marco de referência, cabe neste momento considerar os estudos que previamente tentaram relacionar a responsabilidade com os valores humanos e o individualismo-coletivismo. Certamente nem todos estabelecem esta relação de forma direta, embora seja possível deduzi-la.

\section{RESPONSABILIDADE, VALORES E INDIVIDUALISMO-COLETIVISMO}

Inicialmente cabe destacar que não foi encontrado nenhum estudo no Brasil que considerasse especificamente uma medida de responsabilidade pessoal, relacionando-a com os valores ou o individualismo-coletivismo. No caso de pesquisas em outros países, foi possível identificar uma brevemente descrita por Rokeach (1973). Na realidade esse autor fala em honestidade em lugar de responsabilidade; $\mathrm{O}$ estudo relatado sugere um conjunto específico de valores como explicadores do comportamento honesto, no caso devolver uma caneta que tinha sido emprestada para preencher um questionário. Concretamente, os postos medianos para os valores um mundo em paz, salvação, prestativo e honesto foram estatisticamente diferentes em função dos grupos compostos por pessoas que devolveram a caneta vs. aquelas que não o fizeram. Aqueles que se comportam honestamente deram maior ênfase a estes valores.

Gouveia e cols. (2000) estudaram em que medida o individualismo-coletivismo se correlacionaria com a atribuição de responsabilidade social. Seu estudo foi realizado com uma amostra de estudantes universitários, procurando comprovar o argumento de que alguns atributos de ordem pessoal (atitudes) também podem ser importantes na explicação da responsabilidade social. Para tanto, aplicaram o Questionário de IndividualismoColectivismo (Triandis, Bontempo, Villareal, Asai \& Lucca, 1988) e efetuaram um experimento em que eram descritas situações nas quais os atores se comportavam de modo socialmente não responsável. Os resultados obtidos, independentemente da situação, evidenciaram que a pontuação de individualismo-coletivismo não se correlacionou significativamente com a atribuição de responsabilidade social ( $\underline{r}$ médio $\leq|0,05|)$.

Estes resultados, na realidade, não são tão esclarecedores ou conclusivos. Além de não se referirem, especificamente, à responsabilidade pessoal, não apresentam um marco teórico sólido que permita coerentemente explicá-los. Neste sentido, parece evidente a lacuna referente à compreensão do papel dos valores e do individualismo-coletivismo para explicar este tipo de responsabilidade. Como antes se sugeriu, este é o principal objetivo do presente estudo, cujo método é descrito a seguir.

\section{MÉTODO}

\section{Delineamento e hipótese}

Neste estudo se assumiu um delineamento correlacional, considerando-se como variáveis antecedentes os valores humanos e o individualismocoletivismo e como critério a responsabilidade pessoal. Levando-se em conta o antes comentado, formulou-se a hipótese geral de que as pessoas que apresentam uma orientação mais social/coletivista tenderão a apresentar maior pontuação na medida de responsabilidade pessoal.

\section{Amostra}

Participaram 250 estudantes de Psicologia de uma universidade pública espanhola, a maioria do sexo feminino $(82 \%)$ e solteira $(94,7 \%)$, com idade variando de 17 a 40 anos $(M=20,3 ; D P=2,94)$. Estes 
apresentaram uma média de religiosidade autopercebida de 1,4 (DP = 1,22; amplitude de $\mathbf{0}=$ Nada religioso a $4=$ Muito religioso).

\section{Instrumentos}

Os participantes preencheram as seguintes medidas:

- Questionário dos Valores Básicos: compõe-se de 110 itens, igualmente distribuídos em 22 valores básicos: afetividade, apoio social, autodireção, beleza, conhecimento, convivência, emoção, estabilidade pessoal, estimulação, êxito, honestidade, justiça social, maturidade, ordem social, poder, prestígio, privacidade, religiosidade, saúde, sexual, sobrevivência e tradição. Para responder aos itens o participante deve indicar quanto o considera importante enquanto um princípio-guia na sua vida, utilizando uma escala de resposta com os seguintes extremos: $\mathbf{0}=$ Nada importante e $\mathbf{4}=$ Totalmente importante. Indicadores dos seus parâmetros psicométricos podem ser observados em Gouveia (1998). Por exemplo, este autor encontrou índices de consistência interna (Alfa de Cronbach) variando de 0,56 (estimulação) a 0,96 (religiosidade), com Alfa médio de 0,71.

- Questionário de individualismo-coletivismo: este instrumento foi originalmente proposto por Triandis e cols. (1988), sendo adaptado ao contexto espanhol por Morales, López e Vega (1992). Compõe-se de 29 itens, respondidos em uma escala de resposta de cinco pontos, com os seguintes extremos: $\mathbf{1}=$ Discordo totalmente e $\mathbf{5}=$ Concordo totalmente. No presente estudo se considera sua versão reduzida (Gouveia \& cols., 2000), composta de 12 itens (por exemplo, o êxito é o mais importante na vida; minha felicidade não está relacionada com o bem-estar dos meus companheiros). Esta estrutura unifatorial permitiu explicar $14 \%$ da variância total das respostas dos participantes, tendo apresentado uma consistência interna (Alfa de Cronbach) de 0,73. A maior pontuação neste instrumento corresponde a um maior nível de individualismo, enquanto a menor é indicadora de coletivismo.

- Escala de responsabilidade pessoal: esta medida se compõe de 10 itens que indicam condutas irresponsáveis (por exemplo, faltar a compromissos previamente marcados; prometer algo aos demais e não cumprir). Neste sentido, as pontuações dos participantes precisam ser invertidas para indicar o grau de responsabilidade pessoal. Suas respostas são dadas em escala de cinco pontos, tipo Likert, com os seguintes extremos: $\mathbf{0}=$ Nada característico e $\mathbf{4}=$ Totalmente característico. Gouveia e cols. (2000) apresentam informações sobre seus parâmetros psicométricos. Assinalam, por exemplo, que sete dos seus dez itens apresentam saturação igual ou superior a $|0,40|$, definindo um fator único com Alfa de Cronbach de 0,66 e possibilitando explicar $24,59 \%$ da variância total.

Além das medidas antes descritas, os participantes também responderam a questões de caráter sociodemográfico, úteis para descrever a amostra do estudo (por exemplo, sexo, idade e estado civil).

\section{Procedimento}

Solicitou-se aos participantes sua colaboração para responder aos instrumentos previamente descritos. Estes foram aplicados em sala de aula, com a autorização prévia do professor responsável pela disciplina. Os participantes foram instruídos pelos pesquisadores de que não existiam respostas certas ou erradas, sendo-lhes garantidos o anonimato e a confidencialidade das suas respostas. Com o fim de minimizar os vieses de resposta, os instrumentos foram contrabalanceados, porém deixaram invariavelmente a folha de dados sociodemográficos no final do questionário. Em média, foram necessários 25 minutos para os indivíduos concluírem sua participação no estudo.

\section{RESULTADOS}

\section{Análises preliminares}

Inicialmente é preciso descrever o grau de responsabilidade pessoal dos participantes, como avaliado pela escala antes mencionada. Considerandose o somatório dos sete itens que conformaram esta medida e a escala de resposta empregada, a pontuação teórica mínima (alta responsabilidade) e máxima (baixa responsabilidade) dos participantes são 0 e 28 , respectivamente. As pontuações empíricas respectivas foram: 1 e 23 pontos, com média de 8,7 ( $\mathrm{DP}=4,05$ ). Esta pontuação média é estatisticamente inferior à mediana teórica da escala, situada em 14,5 pontos [ $\mathrm{t}$ $(238)=21,94, \underline{p}<0,001]$. Portanto, considerando-se a direção dos itens desta medida, é possível perceber que os participantes apresentaram condutas que podem ser consideradas responsáveis. Para facilitar a compreensão das análises estatísticas subsequientes, 
decidiu-se inverter a escala de resposta, tornando a máxima pontuação como indicador de maior responsabilidade pessoal.

Em termos dos valores humanos, os participantes deste estudo deram mais importância àqueles que cumprem uma orientação social, especificamente aos interacionais. Três destes valores foram considerados os mais importantes (Tabela 1): afetividade $(\mathrm{M}=3,5, \mathrm{DP}=0,60)$, honestidade $(\mathrm{M}=3,5, \mathrm{DP}=0,50)$ e apoio social $(\mathrm{M}=3,4, \mathrm{DP}=0,59)$. Por outro lado, os três menos importantes foram: religiosidade $(\mathrm{M}=1,3, \mathrm{DP}=$ $1,25)$, poder $(\mathrm{M}=1,6, \mathrm{DP}=0,83)$ e convivência $(\mathrm{M}=1,7, \mathrm{DP}=0,85)$. Embora o primeiro e último valores cumpram uma orientação social, o poder atende a um critério de orientação pessoal. O que todos estes têm em comum é a ênfase no atributo vertical do comportamento, colocando em primeiro lugar o respeito aos padrões culturais e a conformação aos grupos sociais. Este padrão é, portanto, diferente daquele revelado pelos valores mais importantes, cuja ênfase é dada a uma orientação social horizontal.

Finalmente, os participantes apresentaram uma pontuação média de 26,2 pontos ( $\mathrm{DP}=6,37$ ) no fator de individualismo-coletivismo. Lembrando, este se compõe de 12 itens, apresentando as pontuações teóricas no intervalo de 12 a 60 pontos, com mediana de 24,5 pontos. Comparando-se as pontuações teórica e empírica neste construto, comprovou-se que os participantes apresentaram uma orientação mais individualista $[\mathrm{t}(244)=4,45, \mathrm{p}<0,001]$.

Em resumo, a amostra considerada apresenta mais freqüentemente comportamentos de tipo pessoalmente responsável, que prezam valores interacionais, embora apresentem uma tendência ao individualismo. Estes resultados serão posteriormente analisados. De momento, é preciso considerar a relação da responsabilidade pessoal com os valores e o individualismo-coletivismo, interesse principal deste estudo.

\section{Valores, individualismo-coletivismo e responsabilidade}

A hipótese principal do presente estudo previa uma correlação direta entre os valores sociais e o grau de responsabilidade pessoal manifesto pelos participantes. A comprovação desta hipótese se fez através de coeficientes $\underline{r}$ de Pearson, assumindo o critério de teste unicaudal, com eliminação dos dados missing (em branco) através do procedimento pairwise. Os resultados desta análise podem ser vistos na Tabela 1 a seguir.
Tabela 1. Correlação entre os Valores Humanos Básicos e a Responsabilidade Pessoal.

\begin{tabular}{|c|c|c|c|c|}
\hline & \multicolumn{3}{|c|}{ Valores } & \multirow{2}{*}{$\begin{array}{c}\text { Responsabilidade Pessoal } \\
\mathbf{r}\end{array}$} \\
\hline & $\mathbf{M}$ & DP & $\alpha$ & \\
\hline Pessoais & 2,49 & 0,44 & 0,67 & $-0,13 *$ \\
\hline Emoção & 2,10 & 1,01 & 0,84 & $-0,21 * * *$ \\
\hline Poder & 1,59 & 0,83 & 0,78 & $-0,16^{* *}$ \\
\hline Sexual & 2,56 & 0,90 & 0,82 & $-0,13^{*}$ \\
\hline Estimulação & 2,62 & 0,70 & 0,63 & $0,12 *$ \\
\hline Exxito & 3,28 & 0,57 & 0,63 & $0,12^{*}$ \\
\hline Prestígio & 1,95 & 0,84 & 0,64 & $-0,09$ \\
\hline Privacidade & 2,56 & 0,80 & 0,66 & $-0,06$ \\
\hline Autodireção & 3,39 & 0,57 & 0,56 & $-0,04$ \\
\hline Centrais & 2,95 & $\mathbf{0 , 4 0}$ & 0,63 & $0,13 *$ \\
\hline Beleza & 3,20 & 0,71 & 0,78 & $0,14^{*}$ \\
\hline Maturidade & 3,15 & 0,66 & 0,54 & $0,14^{*}$ \\
\hline Justiça social & 3,21 & 0,66 & 0,73 & 0,09 \\
\hline Saúde & 2,76 & 0,75 & 0,60 & 0,06 \\
\hline Estabilidade pessoal & 2,76 & 0,71 & 0,69 & 0,05 \\
\hline Conhecimento & 2,61 & 0,78 & 0,77 & 0,02 \\
\hline Sobrevivência & 3,05 & 0,74 & 0,60 & 0,01 \\
\hline Sociais & 2,65 & 0,47 & $\mathbf{0 , 7 3}$ & $0,18 * *$ \\
\hline Honestidade & 3,51 & 0,50 & 0,68 & $0,33 * * *$ \\
\hline Tradição & 2,06 & 0,77 & 0,72 & $0,17 * *$ \\
\hline Ordem & 2,72 & 0,83 & 0,77 & $0,16^{*}$ \\
\hline Convivência & 1,74 & 0,85 & 0,63 & $0,11^{*}$ \\
\hline Afetividade & 3,52 & 0,60 & 0,57 & 0,07 \\
\hline Apoio social & 3,43 & 0,59 & 0,65 & 0,05 \\
\hline Religiosidade & 1,36 & 1,25 & 0,96 & 0,01 \\
\hline
\end{tabular}

De acordo com a tabela anterior, confirma-se a hipótese deste estudo. Especificamente, observa-se que a pontuação total dos valores sociais, isto é, o somatório dos sete valores que conformam este critério de orientação (afetividade, apoio social, convivência, honestidade, ordem social, religiosidade e tradição) se correlacionou com o sentido e com a magnitude esperada $(\mathrm{r}=0,18, \mathrm{p}<0,001)$. Considerando-se seus valores específicos, constatouse que quatro deles também se correlacionaram diretamente com este construto, como segue: honestidade $(\mathrm{r}=0,33, \mathrm{p}<0,001)$, tradição $(\mathrm{r}=0,17, \mathrm{p}$ $<0,01)$, ordem social $(\mathrm{r}=0,16, \mathrm{p}<0,05) \mathrm{e}$ convivência $(\mathrm{r}=0,11, \mathrm{p}<0,05)$.

Apesar de não ter sido formulada nenhuma hipótese em relação aos valores pessoais, observou-se que a pontuação total destes, que incluem oito valores específicos (autodireção, emoção, estimulação, êxito, poder, prestígio, privacidade e sexual), correlacionouse direta e significativamente com a pontuação de responsabilidade pessoal: $\mathrm{r}=-0,13$ ( $\mathrm{p}<0,05)$. Do total de valores específicos, cinco apresentaram correlações estatisticamente significativas, embora não todas na mesma direção, como segue: emoção $(\mathrm{r}=$ $0,21, \mathrm{p}<0,001)$, poder $(\mathrm{r}=-0,16, \mathrm{p}<0,01)$, sexual 
$(\mathrm{r}=-0,13, p<0,05)$, estimulação e êxito $(\underline{\mathrm{r}}=0,12, \underline{\mathrm{p}}<$ 0,05 ; para ambos).

A pontuação total dos valores centrais, que incluem beleza, conhecimento, estabilidade pessoal, justiça social, maturidade, saúde e sobrevivência, correlacionou-se direta e significativamente com a responsabilidade pessoal $(\mathrm{r}=0,13, \mathrm{p}<0,05)$. No caso dos seus valores específicos, dois apresentaram o mesmo padrão de correlação: beleza e maturidade $(\mathrm{r}=$ $0,14, \mathrm{p}<0,05)$.

Além dos resultados acima, considerou-se a possibilidade de compreender a responsabilidade pessoal a partir das funções psicossocias dos valores humanos, como listadas na Figura 1.

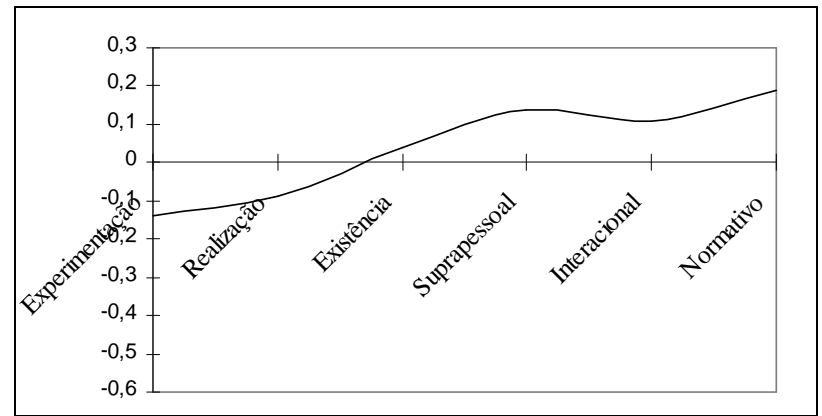

Figura 1. Correlação entre responsabilidade pessoal e as funções psicosociais dos valores humanos.

De acordo com esta figura, comprova-se a existência de uma curva sinusoidal entre as funções psicossociais dos valores e a responsabilidade pessoal, isto é, esta correlação é inversa no caso da função de experimentação $(\mathrm{r}=-0,14, \mathrm{p}<0,05)$, sendo nula para a função de existência $(\mathrm{r}=0,04, \mathrm{p}>0,05)$ e direta no caso da normativa $(\mathrm{r}=0,19, \mathrm{p}<0,01)$. A função realização apresentou uma correlação inversa com a responsabilidade pessoal $(\mathrm{r}=-0,09, \mathrm{p}<0,05)$ e uma correlação direta com as funções suprapessoal $(r=$ $0,14, p<0,05)$ e interacional $(\mathrm{r}=0,11, \mathrm{p}<0,05)$.

Por fim, com o fito de conhecer a extensão da dimensão social da responsabilidade pessoal, procurou-se correlacionar sua pontuação com a da medida de individualismo-coletivismo. A propósito, observou-se uma correlação inversa e significativa $(\mathrm{r}=$ $-0,19, \mathrm{p}<0,01)$ entre tais construtos, sugerindo que quem obtém pontuação alta em individualismo apresenta baixa responsabilidade pessoal.

\section{DISCUSSÃO E CONCLUSÃO}

O objetivo do presente estudo, como ficou evidenciado, foi conhecer em que medida a responsabilidade pessoal estaria correlacionada com os valores humanos e o individualismo-coletivismo. Espera-se que este tenha sido cumprido. Não obstante, é impossível deixar de assinalar ao menos duas eventuais limitações desta pesquisa: (1) o fator principal de responsabilidade pessoal reúne poucos itens e apresenta um índice de consistência interna apenas meritório (Alfa $=0,66$ ). É possível que esta limitação psicométrica se reflita nas correlações baixas $(\underline{\mathrm{r}} \leq|0,33|)$ observadas. Portanto, recomenda-se construir uma nova medida deste construto ou, se for o caso, validar alguma existente em outra cultura; e (2) a amostra considerou unicamente estudantes universitários. Estes costumam ter uma orientação social horizontal, primando por valores como justiça social. Talvez este aspecto influencie a pontuação dos participantes nas dimensões de responsabilidade social e individualismo-coletivismo. Portanto, para garantir a generalização dos resultados aqui apresentados, sugere-se replicar este estudo considerando-se participantes da população geral.

Em termos específicos dos resultados, cabe destacar, reforçando o anteriormente descrito, que os participantes se mostraram pessoalmente responsáveis, certamente por incorporar a educação formal recebida na Universidade. Estes podem ser também descritos como mais tendentes ao individualismo, apesar da maior importância dada aos valores que atendem à função psicossocial interacional. Este padrão claramente define a cultura espanhola, que ressalta o envolvimento leal com os membros do endogrupo (Smith, Dugan \& Trompenaars, 1996), embora encontre soluções individualistas para alguns problemas do seu dia-a-dia (Andrés-Orizo, 1996).

A respeito do individualismo e coletivismo na Espanha, um estudo recente de Gouveia e cols. (2003) reforça a idiossincrasia da orientação social dos espanhóis. Mais do que serem classificados como individualistas ou coletivistas, estes podem ser melhor definidos como orientados ao princípio de igualdade e/ou justiça social, como manifesto na sua pontuação tanto no individualismo horizontal $(\mathrm{M}=52,3, \mathrm{DP}=$ 8,42; por exemplo, freqüentemente faço minhas próprias coisas; sou uma pessoa única) como no coletivismo horizontal $(\mathrm{M}=54,8, \mathrm{DP}=9,14$; por exemplo, é importante para mim o bem-estar dos meus companheiros de trabalho; para mim, prazer é passar o tempo com os demais).

Quanto à hipótese geral aqui anunciada, é evidente sua corroboração. O padrão de correlação da responsabilidade pessoal com os valores sociais foi estatisticamente significativo e na direção esperada, isto é, estão correlacionados diretamente. Quanto maior a importância atribuída aos valores sociais, mais 
responsabilidade a pessoa indica manifestar. Esta situação é especialmente válida para quem se pauta pelo valor honestidade, mas não menos para aqueles guiados pelos valores tradição, ordem social e convivência. Isso evidencia que, ao menos na cultura espanhola, apresentar responsabilidade social implica um compromisso com os demais e o cumprimento das normas sociais vigentes. Estes são, provavelmente, valores de maior peso para explicar a responsabilidade das pessoas em culturas coletivistas ou relacionais (Triandis, 1995). Neste contexto, provavelmente as pessoas se sintam "obrigadas" a se comportar responsavelmente, evitando defraudar aqueles com os quais mantêm interação. A propósito, seria conveniente avaliar em pesquisa futura os fatores que levam as pessoas a se comportarem de forma pessoalmente responsável. A explicação mais plausível teria uma natureza intrínseca ou extrínseca às pessoas?

Reforçando a natureza social da responsabilidade pessoal, comprovou-se que, no conjunto, os valores pessoais e o individualismo se correlacionaram negativamente com este construto. Este padrão de correlação ficou especialmente evidente para os valores de experimentação, especificamente emoção e sexual. Pessoas que se pautam por estes valores, de acordo com o padrão de personalidade correspondente, relevam-se como buscadores de sensação, estando mais vulneráveis ao uso potencial de drogas (Coelho Júnior, 2001) e a apresentar comportamentos anti-sociais (Formiga, 2002). Portanto, é coerente conjeturar que estas pessoas comumente apresentam comportamentos pessoalmente irresponsáveis.

Finalmente, sugere-se replicar esta pesquisa no contexto brasileiro, incluindo também pessoas da população geral. É possível que os resultados principais se revelem consistentes, porém é esperada uma maior força explicativa dos valores de natureza normativa, visto que este padrão de orientação é bastante comum no Brasil, especialmente na Região Nordeste (Gouveia, 1998). Este país, definido geralmente como coletivista (Gouveia e cols., 2003; Triandis, 1995), pode favorecer dados que permitam compreender em maior medida a dinâmica da responsabilidade pessoal quando o comportamento do indivíduo pode ter repercussão direta sobre a percepção e o autoconceito dos membros do seu grupo de pertença.

\section{REFERÊNCIAS}

Andrés-Orizo, F. (1996). Sistemas de valores en la España de los 90. Madri: Centro de Investigaciones Sociológicas.
Braithwaite, V. \& Scott, W. A. (1991). Values. Em J. P. Robinson, P. R. Shaver \& L. S. Wrightsman (Eds.), Measuring of personality and social psychological attitudes, (Vol. 1, pp. 661-753). San Diego, CA: Academic Press.

Coelho Júnior, L. L. (2001). Uso potencial de drogas em estudantes do ensino médio: sua correlação com as prioridades axiológicas. Dissertação de Mestrado, Universidade Federal da Paraíba, João Pessoa.

Formiga, N. S. (2002). Condutas anti-sociais e delitivas: uma explicação baseada nos valores humanos. Dissertação de Mestrado. Departamento de Psicologia, Universidade Federal da Paraíba, João Pessoa.

Gouveia, V.V. (1998). La naturaleza de los valores descriptores del individualismo y del colectivismo: una comparación intra e intercultural. Tese de Doutorado, Universidade Complutense de Madri, Espanha.

Gouveia, V. V., Albuquerque, F. J. B., Clemente, M. \& Espinosa, P. (2003). The horizontal and vertical attributes of individualism and collectivism in a Spanish population. The Journal of Social Psychology, 143, 43-63.

Gouveia, V. V. \& Alves, S. M. (1993). Tipos de comportamento e proximidade ator-observador: suas influências na atribuição de responsabilidade social. Psicologia: Reflexão e Crítica, 6, 49-56

Gouveia, V.V., Andrade, J.M., Milfont, T. L. \& Santos, W. S. (2003). Dimensões normativas do individualism e coletivismo: é suficiente a dicotomia pessoal vs. Social? Psicologia: Reflexão e Crítica, 16, 223-234.

Gouveia, V.V., Clemente, M. \& Vidal, M. A. (1998). España desde dentro: el individualism y el colectivismo como rasgos diferenciadores de las Comunidades Autónomas. Sociedad y Utopia. Revista de Ciencias Sociales, 11, 167179.

Gouveia, V. V., Clemente, M., Vidal, M. A. \& Martinez, E. (2000). Atribución de responsabilidad social: contexto social y atributos personales del observador. Revista de Psicología Social, 15, 41-51.

Hofstede, G. (1984). Culture's consequences: international differences in work-related values. Beverly Hills, CA: Sage Publications.

Maslow, A. H. (1954). Motivation and personality. New York: Harper \& Row Publishers.

Morales, J. F., López, M. \& Vega, L. (1992). Individualismo, colectivismo e identidad social. Revista de Psicología Social, Monográfico, 49-72.

O’Neill, P. \& Eisner, M. (1981). Responsibility and decisionmaking: factors that contribute to careful attention in choice task. Canadian Journal of Behavioral Science, 13, 288-296.

Rokeach, M. (1973). The nature of human values. New York: The Free Press.

Schwartz, S. H. (1994). Are there universal aspects in the structure and contents of human values? Journal of Social Issues, 50, 19-45.

Sigelmann, E. \& Fernandes, L. M. (1984). A medida da responsabilidade social. Arquivos Brasileiros de Psicologia, 4,26-41.

Smith, P. B., Dugan, S. \& Trompenaars, F. (1996). National culture and the values of organizational employees: a 
dimensional analysis across 43 nations. Journal of CrossCultural Psychology, 27, 231-264.

Triandis, H. C. (1994). Culture and social behavior. New York: McGraw-Hill.

Triandis, H. C. (1995). Individualism and collectivism. Boulder, CO: Westview Press.

Triandis, H. C., Bontempo, R., Villareal, M. J., Asai, M. \& Lucca, N. (1988). Individualism and collectivism: Crosscultural perspectives on self-ingroup relationships. Journal of Personality and Social Psychology, 54, 323-338.
Waterman, A. S. (1981). Individualism and interdependence. American Psychologist, 7, 762-773.

Endereço para correspondência: Valdiney Veloso Gouveia, Rua Hortêncio Osterne Carneiro, 598, Bessa, CEP 58035-120, João Pessoa-PB. E-mail: vvgouveia@uol.com.br 